\title{
Heme Modification Contributes to the Mechanism-Based Inactivation of Human Cytochrome P450 2J2 by Two Terminal Acetylenic Compounds
}

\author{
Hsia-lien Lin, Haoming Zhang, Vyvyca J. Walker, Jaime D'Agostino, and Paul F. Hollenberg \\ Department of Pharmacology, University of Michigan, Ann Arbor, Michigan
}

Received March 9, 2017; accepted July 6, 2017

\section{ABSTRACT}

The mechanism-based inactivation of human CYP2J2 by three terminal acetylenic compounds: $\boldsymbol{N}$-(methylsulfonyl)-6-(2-propargyloxyphenyl)hexanamide (MS), 17-octadecynoic acid (OD), and danazol (DZ) was investigated. The loss of hydroxyebastine (OHEB) carboxylation activity in a reconstituted system was time- and concentration-dependent and required NADPH for MS and OD, but not $D Z$. The kinetic constants for the mechanism-based inactivation of OHEB carboxylation activity were: $K_{1}$ of $6.1 \mu \mathrm{M}$ and $k_{\text {inact }}$ of $0.22 \mathrm{~min}^{-1}$ for MS and $K_{1}$ of $2.5 \mu \mathrm{M}$ and $k_{\text {inact }}$ of $0.05 \mathrm{~min}^{-1}$ for OD. The partition ratios for MS and OD were $\sim 10$ and $\sim 20$, respectively. Inactivation of CYP2J2 by MS or OD resulted in a loss of the native heme spectrum and a similar decrease in the reduced CO difference spectrum. A heme adduct was observed in the
MS-inactivated CYP2J2. The possible reactive metabolite which covalently modified the prosthetic heme was characterized by analysis of the glutathione conjugates formed by MS or OD following oxygenation of the ethynyl moiety. Liquid chromatography-mass spectrometry showed that inactivation by MS or OD did not lead to modification of apoprotein. Interaction of CYP2J2 with DZ produced a type II binding spectrum with a $K_{\mathrm{s}}$ of $2.8 \mu \mathrm{M}$ and the $\mathrm{IC}_{50}$ for loss of OHEB carboxylation activity was $0.18 \mu \mathrm{M}$. In conclusion, heme modification by MS and OD was responsible for the mechanismbased inactivation of CYP2J2. The results suggest that the ethynyl moiety of MS and OD faces the heme iron, whereas the isoxazole ring of DZ is preferentially oriented toward the heme iron of CYP2J2.

\section{Introduction}

Cytochrome P450 2J2 (CYP2J2), a member of the human cytochrome P450 family, is distributed in the heart, lungs, intestines, liver, kidneys, brain, and in some tumor tissues (Wu et al., 1996; Zeldin et al., 1996, 1997; Jiang et al., 2005; Dutheil et al., 2009). It is one of the primary $\mathrm{P} 450$ s responsible for the biotransformation of arachidonic acid (AA) to four regioisomeric epoxyeicosatrienoic acids (EETs) (5,6-, 8,9-, 11,12-, and 14,15-EET) which are biologically active (Wu et al., 1996; Chaudhary et al., 2009; Jenkins et al., 2009). EETs have important functions in the regulation of cellular proliferation, blood pressure, hormone release, inflammatory response, and various cellular signal pathways relevant to cardiovascular and renal functions and in protecting endothelial cells from hypoxia-reoxygenation injury ( $\mathrm{Wu}$ et al., 1996; Zeldin et al., 1996, 1997; Node et al., 1999; Yang et al., 2001; Fleming and Busse, 2006; Spector and Norris, 2007). Because CYP2J2 is mainly expressed in extrahepatic tissues, including the heart and intestines, and its AA metabolites have important pathologic and physiologic properties, interest in CYP2J2 has grown significantly in the last 10 years. Recent studies include: 1) the role of EETs in protecting the myocardium following ischemia/reperfusion injury and the role of EET administration or CYP2J2 over-expression in protecting rat heart against tumor necrosis factor- $\alpha$-induced injury; (Seubert et al., 2007;

This work was supported in part by National Institutes of Health National Cancer Institute [Grant R01 CA-16954] (to P.F.H.).

https://doi.org/10.1124/dmd.117.075846.
Zhao et al., 2012); 2) the identification of novel substrates or clinical drugs for human cytochrome P450 2J2 (Lee et al., 2010; Wu et al., 2013); and 3) the identification of selective inhibitors of CYP2J2 (Lafite et al., 2006, 2007a; Chen et al., 2009; Lee et al., 2012; Ren et al., 2013).

The terminal acetylenic compounds, 17-octadecynoic acid (OD) and $\mathrm{N}$-methylsulfonyl-6-(2-propargyloxyphenyl)hexanamide (MS), were designed to be selective inhibitors of the P450-catalyzed metabolism of AA (Zou et al., 1994; Wang et al., 1998; Brand-Schieber et al., 2000). Their inhibitory effects on members of P450 4A family were time- and NADPHdependent, suggesting that OD and MS functioned as mechanism-based inactivators. However, studies of the kinetic values, the partition ratios, and the molecular mechanisms responsible for the mechanism-based inactivation have not been reported. Recently, MS and OD were used as selective inhibitors to investigate: 1 ) the possible role of CYP2J2 epoxygenase in the proliferation of tumor cells (Jiang et al., 2009); 2) CYP2J2 as a novel biomarker and potential target for therapy of human cancer (Jiang et al., 2005); and 3) inhibition of carcinoma cell motility by EET antagonists (Nithipatikom et al., 2010). In a separate study, perfusion of mouse hearts with MS before ischema/reperfusion abolished the improved postischemic recovery observed in CYP2J2 transgenic mice (Seubert et al., 2004). In addition, danazol (DZ) was identified as a selective and potent inhibitor for assessing the role of CYP2J2 in drug metabolism (Lee et al., 2012). DZ is a synthetic androgen that contains a terminal ethynyl moiety. Since all three inhibitors have been widely used for studies involving CYP2J2, it was of interest to characterize the abilities of MS, OD, and DZ to act as timedependent or mechanism-based inactivators of CYP2J2.

ABBREVIATIONS: CYP2J2, human cytochrome P450 2J2; GSH, glutathione; CPR, NADPH-cytochrome P450 reductase; HPLC, high-pressure liquid chromatography; EB, ebastine; OHEB, hydroxyebastine; CEB, carebastine; DZ, danazol (4-androsten-[2,3-d]isoxazole-17 $\alpha$-ethynyl-17 $\beta$-ol); MS, N-methylsulfonyl-6-(2-propargyloxyphenyl)hexanamide; OD, 17-octadecynoic acid; TFA, trifluoroacetic acid; ESI, electrospray ionization; LCMS, liquid chromatography-mass spectrometry; LC-MS/MS, liquid chromatography-tandem mass spectrometry; $K_{\mathrm{s}}$, spectral dissociation constant. 
A

$\mathrm{N}$-methylsulfonyl-6-(2-propargyloxyphenyl)hexanamide (MS)<smiles>C#CCOc1ccccc1CCCCCC(=O)N(C)S(C)(=O)=O</smiles>

17-Octadecynoic acid (OD)

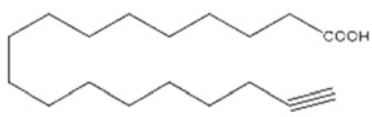

Danazol (DZ)<smiles>C#C[C@]1(O)CCC2C3CCC4=Cc5oncc5CC4(C)C3CC[C@]21C</smiles>

B

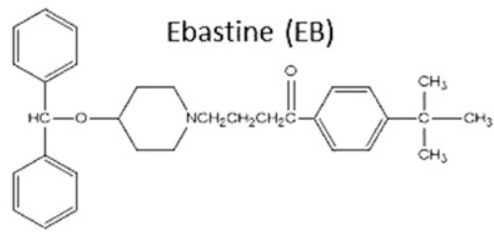

Hydroxyebastine (OHEB)

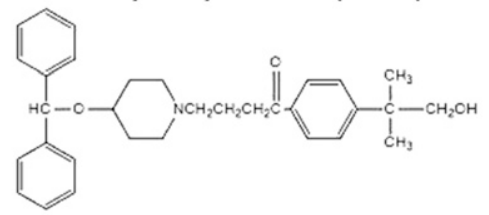

Carebastine (CEB)

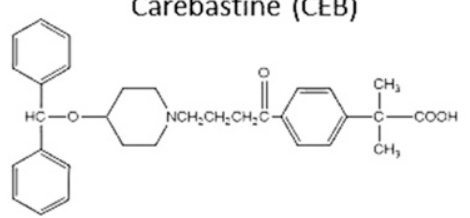

Fig. 1. Chemical structures of compounds characterized in this study. (A) the three acetylenes: MS, OD and DZ. (B) prodrug EB and its two metabolites OHEB and CEB.
Ebastine (EB), a potent nonsedative H1-antihistamine, has two major pathways for metabolism: hydroxylation and $N$-dealkylation (Hashizume et al., 1998, 2002). CYP2J2 has been shown to play an important role in EB metabolism. The hydroxylation of EB to give the intermediate hydroxyebastine (OHEB) followed by the conversion of OHEB to the pharmacologicaly active carebastine (CEB) are preferentially catalyzed by CYP2J2 (Hashizume et al., 2002; Liu et al., 2006). In contrast, CYP3A4-dependent dealkylation of EB, OHEB and CEB leads to the formation of the inactive metabolite dealkylebastine (Hashizume et al., 2002; Liu et al., 2006). Purified human CYP2J2 reconstitued with NADPH-cytochrome $\mathrm{P} 450$ reductase (CPR) and lipids was used in this study. Initially, we chose EB as the probe substrate for the CYP2J2 catalytic activity (Hashizume et al., 2002; Matsumoto et al., 2002). However, during the course of our studies on EB metabolism using highpressure liquid chromatography (HPLC), we observed that the OHEB product underwent further metabolism to CEB after 5 minute of incubation. To avoid the complication of subsequent metabolism of the EB product, we decided to use OHEB as the substrate probe for characterizing the mechanism-based inactivation of CYP2J2 by MS, OD and DZ.

We report here that MS and OD, but not DZ, are mechanism-based inactivators of CYP2J2 as assessed by measuring OHEB carboxylation to form CEB. The mechanism for the inactivation is heme modification as evidenced by the loss of the native heme, reduced $\mathrm{CO}$ spectra, and formation of a heme adduct during the inactivation of CYP2J2 by MS.

\section{Materials and Methods}

Chemicals. NADPH, glutathione (GSH), chloramphenicol, and catalase were purchased from Sigma-Aldrich (St. Louis, MO). Carbenicillin was from RPI Research Products International Corp. (Mt. Prospect, IL). EB, OHEB, and CEB were from Santa Cruz Biotechnology (Santa Cruz, CA). DZ, MS, and OD were from Cayman Chemical Company (Ann Arbor, MI). The chemical structures of the three acetylenic compounds: MS, OD and DZ as well as prodrug EB, intermediate metabolite OHEB and active metabolite CEB are displayed in Fig. 1. All other chemicals and solvents were of the highest purity available from commercial sources.
Enzyme Purification. CYP2J2 was expressed with some modifications as described previously (Smith et al., 2008). The CYP2J2 cDNA construct was a generous gift from Dr. Rheem Totah at the University of Washington (Seattle, WA). Briefly, the CYP2J2 plasmid was transformed into Escherichia coli C41 competent cells that had been initially transformed with the pGRO7 expression plasmid (Takara Bio Inc., Shiga, Japan). LB plates treated with carbenicillin $(100 \mu \mathrm{g} / \mathrm{ml})$ and chloramphenicol $(40 \mu \mathrm{g} / \mathrm{ml})$ were used to select for bacteria which co-expressed the CYP2J2 construct and pGRO7 plasmid. The purification of CYP2J2 was performed as described previously for the purification of members of CYP2B family (Scott et al., 2001). CPR was expressed in Escherichia coli TOPP 3 cells and purified according to previously published procedures (Lin et al., 2002).

Catalytic Activity and Inactivation. To assess catalytic activity, the purified CYP2J2, lipid, and CPR were reconstituted at $22^{\circ} \mathrm{C}$ for 45 minute. The primary reaction mixture contained $1 \mathrm{nmol} \mathrm{P} 450,2 \mathrm{nmol} \mathrm{CPR}, 60 \mu \mathrm{g}$ of a mixture $(1: 1: 1)$ of L- $\alpha$-dilauroyl-phosphatidylcholine, L- $\alpha$-dioleyl-sn-glycero-3-phosphatidylcholine and L- $\alpha$-phosphatylserine, $2 \mathrm{mM} \mathrm{GSH}, 100$ units of catalase, and OD or MS (1-100 $\mu \mathrm{M})$ in $1 \mathrm{ml}$ of $100 \mathrm{mM}$ potassium phosphate buffer (pH 7.7). After incubation of the primary reaction mixture in the presence of $1 \mathrm{mM} \mathrm{NADPH}$ at $37^{\circ} \mathrm{C}$ with $\mathrm{OD}$ or MS at the concentrations indicated and for the times indicated, a $5 \mu \mathrm{l}$ aliquot was removed and added to $250 \mu \mathrm{l}$ of a secondary reaction mixture containing $20 \mu \mathrm{M}$ OHEB and $200 \mu \mathrm{M}$ NADPH and incubated at $37^{\circ} \mathrm{C}$ for 20 minute. The reaction was stopped by adding $1 \mathrm{ml}$ of acetonitrile containing $0.2 \mathrm{nmol}$ of $16 \beta$-hydroxytestosterone as the internal standard. After centrifugation at $16,000 \mathrm{~g}$ for 10 minute, $1 \mathrm{ml}$ of the supernatant was transferred to a glass tube, dried down under $\mathrm{N}_{2}$ and the residue dissolved in $130 \mu$ lof $50 \%$ methanol for analysis by HPLC. An aliquot $(100 \mu \mathrm{l})$ was injected onto a C8 column (Zorbax XDB, $5 \mu \mathrm{m}, 4.6 \times 250 \mathrm{~mm}$; Agilent Technologies, Santa Clara, CA). The samples were separated isocratically using $60 \%$ of $12 \mathrm{mM}$ ammonium acetate buffer ( $\mathrm{pH} 4.5$ ) in acetonitrile (40\%) at a flow rate of $1 \mathrm{ml} / \mathrm{min}$ for 20 minute. The eluates were monitored by UV detection at $254 \mathrm{~nm}$ (Hashizume et al., 2002; Matsumoto et al., 2002). The metabolite, CEB, and the internal standard were quantified and the data were analyzed for the time- and concentration-dependent loss of activity. Calculations of the kinetic values for mechanism-based inactivation were performed as previously described (Lin et al., 2002, 2013). Each experiment was repeated at least twice and done in triplicate with the replicates differing by no more than $10 \%$. The goodness of fit for all experiments in which regression analysis was used had the $r^{2}>0.95$.

Determination of the Partition Ratio. MS or OD at concentrations ranging from 1 to $200 \mu \mathrm{M}$ were added to primary reaction mixtures containing $1 \mu \mathrm{M}$ 


\section{A Metabolism of EB by CYP2J2 B Kinetics for OHEB metabolism by CYP2J2}
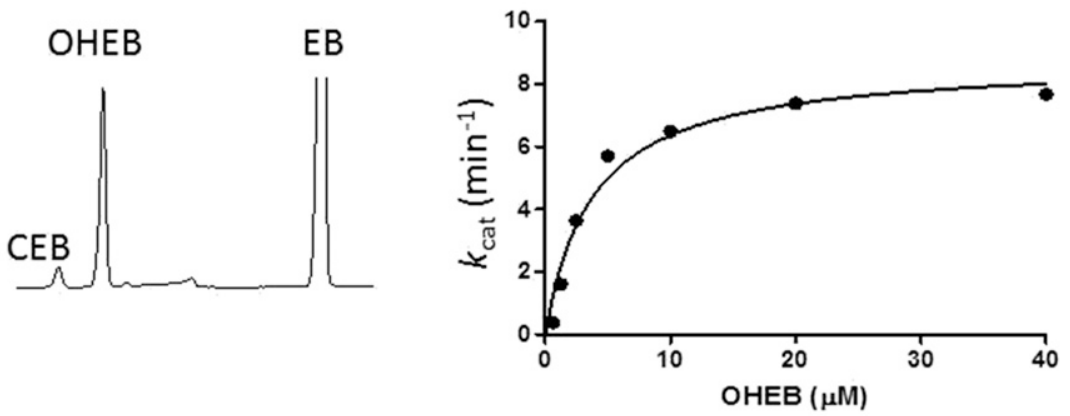

C Metabolism of OHEB by CYP2J2 and CYP3A4

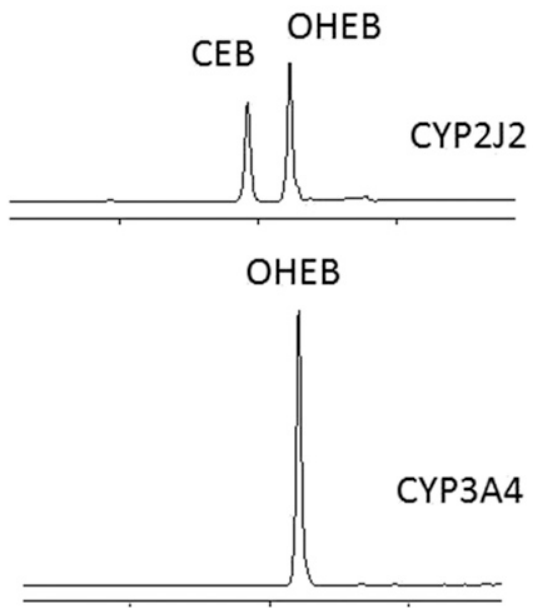

CYP2J2. The reactions were initiated by the addition of $1 \mathrm{mM}$ NADPH and incubated for 30 minute to allow the inactivation to go to completion (Silverman, 1996). Aliquots were removed and assayed for residual catalytic activity as described above and the data analyzed as described previously (Lin et al., 2002).

Spectral and HPLC Analysis. After incubating the primary reaction mixture with $50 \mu \mathrm{M} \mathrm{MS}$ or $\mathrm{OD}$ at $37^{\circ} \mathrm{C}$ for 10 minute, the reduced $\mathrm{CO}$ difference spectra of the control (-NADPH) or inactivated (+NADPH) CYP2J2 (200 pmol) were determined by scanning from 400 to $500 \mathrm{~nm}$ on a UV-2501PC spectrophotometer (Shimadzu, Kyoto, Japan) as described previously (Lin et al., 2002). An HPLC system with a Waters $600 \mathrm{E}$ system controller was used to investigate the loss of native heme and the formation of heme adducts. Aliquots containing $100 \mathrm{pmol}$ of control or inactivated CYP2J2 were analyzed using a C4 reverse-phase column ( $5 \mu \mathrm{m}, 4.6 \times 250 \mathrm{~mm}, 300 \AA \AA$; Phenomenex, Torrance, CA). The solvent system consisted of solvent A ( $0.1 \%$ trifluoroacetic acid [TFA] in water) and solvent B ( $0.1 \%$ TFA in acetonitrile). The column was eluted with a linear gradient from $35 \%$ to $80 \%$ B over 30 minute at a flow rate of $1 \mathrm{ml} / \mathrm{min}$ and the eluate was monitored for heme at $400 \mathrm{~nm}$. The absorption spectra of the native and MS-modified heme were determined using a model 996 photodiode-array Detector (Millipore Corporation, Bilerica, MA).

ESI-LC-MS (Electrospray-Liquid Chromatography-Mass Spectrometry) Analysis of the Apoprotein. Control and inactivated samples incubated with $50 \mu \mathrm{M}$ OD or MS for 10 minute were prepared as described above. Aliquots containing 50 pmol of $\mathrm{P} 450$ were analyzed on a $\mathrm{C} 3$ reverse-phase column (Zorbax 300SB-C3, $3.5 \mu \mathrm{m}, 3.0 \times 150 \mathrm{~mm}$; Agilent Technologies) equilibrated with 35\% acetonitrile and $0.1 \%$ TFA in water at a flow rate $0.2 \mathrm{ml} / \mathrm{min}$. After 5 minute, the column effluent was directed into the mass analyzer of a LCQ mass spectrometer (Thermo Fisher Scientific, San Jose, CA) as described previously (Kent et al.,
Fig. 2. Metabolism of EB and OHEB by CYP2J2. (A) HPLC elution profile for the metabolites formed from EB in the CYP2J2 reconstituted system incubated at $37^{\circ} \mathrm{C}$ for 5 minute. (B) Kinetics for the formation of CEB from OHEB. The CYP2J2 reconstituted systems were incubated with increasing concentrations of OHEB from 0.625 to $40 \mu \mathrm{M}$ at $37^{\circ} \mathrm{C}$ for 10 minute. The data for $k_{\text {cat }}$ versus the OHEB concentrations were fitted to the Michaelis-Menten equation as described under Materials and Methods. (C) HPLC elution profile for the formation of CEB from OHEB by CYP2J2 or CYP3A4 in the reconstituted system following incubation at $37^{\circ} \mathrm{C}$ for 30 minute.
2006; Lin et al., 2009). The acetonitrile concentration was increased linearly to $90 \%$ over the next 25 minute to separate the components of the incubation mixture and the mass spectra were recorded. The spectra corresponding to the protein envelopes for the P450s were deconvoluted to give the masses associated with each protein envelope using the Bioworks software package (Thermo Fisher Scientific). The ESI source conditions were as follows: the sheath gas was set at 90 arbitrary units, the auxiliary gas was set at 30 arbitrary units, the spray voltage was $4.2 \mathrm{kV}$, and the capillary temperature was $230^{\circ} \mathrm{C}$.

Inhibition by DZ. For the inhibition studies, $20 \mu \mathrm{M}$ OHEB was incubated with the CYP2J2 reconstituted system and increasing concentrations of DZ $(0-4 \mu \mathrm{M})$ for 5 minute. The reactions were initiated by adding $200 \mu \mathrm{M}$ NADPH and formation of $\mathrm{CEB}$ was measured following 20 minute incubation at $37^{\circ} \mathrm{C}$ as described above.

Spectral Binding of DZ to CYP2J2. For the binding of DZ to CYP2J2, the spectral dissociation constant $\left(K_{\mathrm{s}}\right)$ was determined by difference spectra on a Shimadzu 2501PC spectrophotometer (Kyoto, Japan). Equal volumes of $1 \mu \mathrm{M}$ solutions of CYP2J2 were added into the reference and the sample cuvette and the baseline recorded. The difference in the absorbance was recorded from 350 to $500 \mathrm{~nm}$ after the addition of a series of aliquots of DZ in methanol to the sample cuvette with the same amount of methanol being added to the reference cuvette. The differences in the absorbance between $\sim 428$ and $\sim 407 \mathrm{~nm}$ at various concentrations of added DZ $(1-22 \mu \mathrm{M})$ were fitted with the Michaelis-Menten equation using GraphPad Prism5 software (San Diego, CA) to obtain the $K_{\mathrm{s}}$ value.

LC-MS/MS Analysis of the GSH Conjugates Formed During Metabolism of MS and OD by CYP2J2. Samples containing 250 pmol of CYP2J2 in $250 \mu \mathrm{l}$ of the reconstituted system reaction mixture were prepared as described above. The samples were incubated with $50 \mu \mathrm{M}$ MS or OD with/without $1 \mathrm{mM}$ 
A

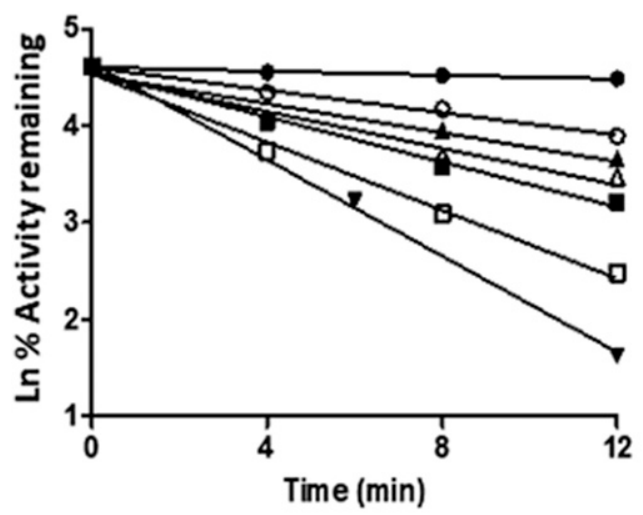

Data 1/MS
B

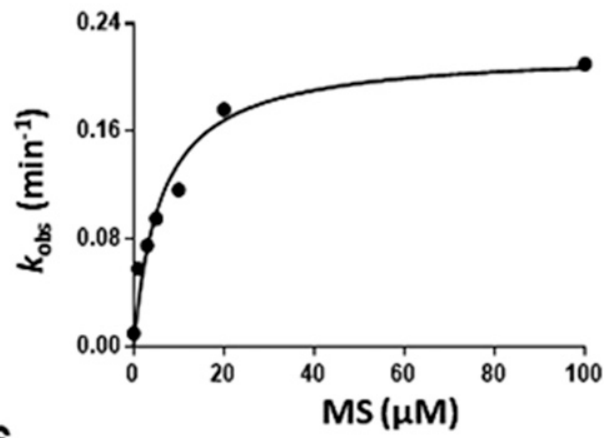

C

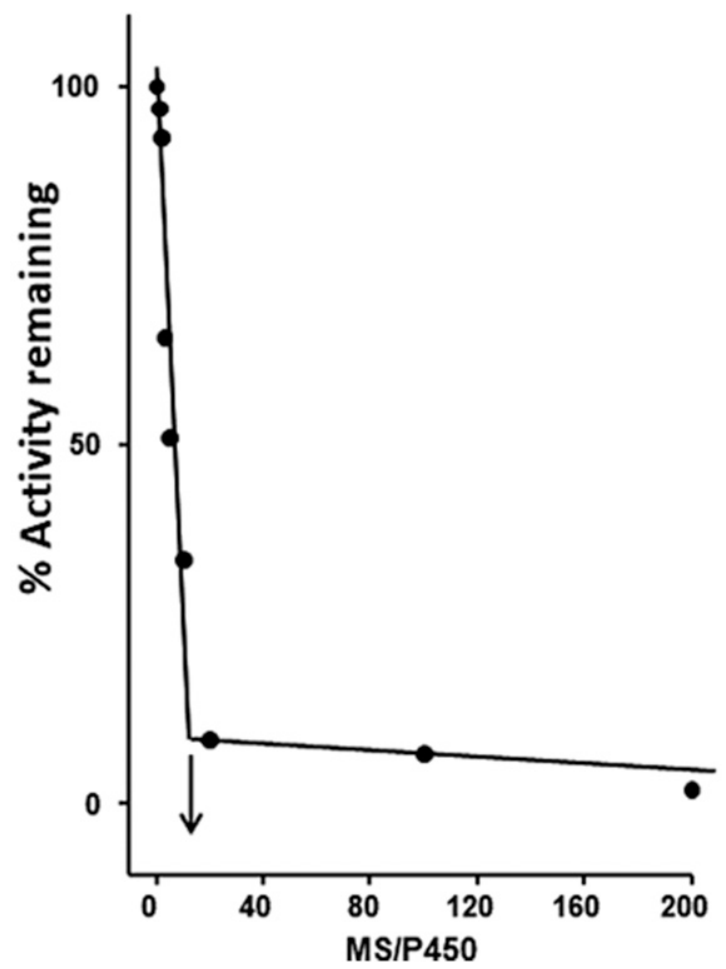

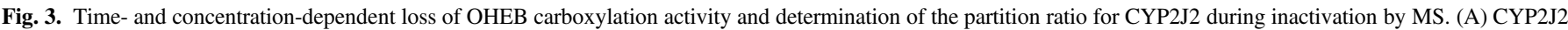

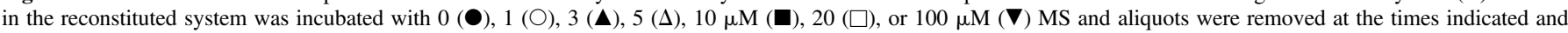

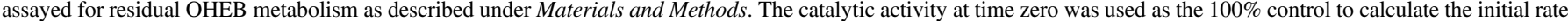

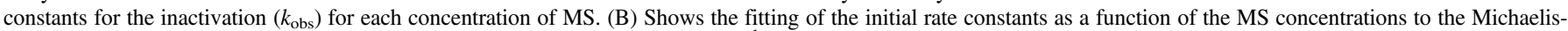

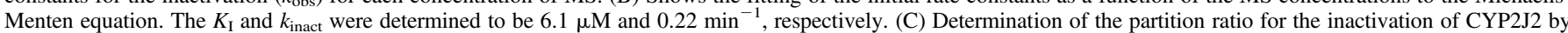

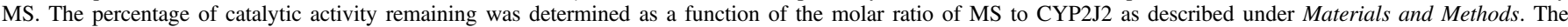

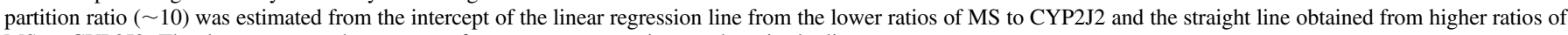
MS to CYP2J2. The data represent the average of two separate experiments done in duplicate.

$\mathrm{NADPH}$ at $37^{\circ} \mathrm{C}$ for 20 minute and then terminated by adding $1 \mathrm{ml}$ of acetonitrile. The mixtures were centrifuged at $13,200 \mathrm{~g}$ at room temperature for 10 minute. The supernatants were dried under $\mathrm{N}_{2}$ gas and re-suspended in $100 \mu \mathrm{l}$ of $50 \%$ methanol. The samples were analyzed on a C18 reverse-phase column (Luna, $3 \mu \mathrm{m}, 4.6 \times$ $100 \mathrm{~mm}$; Phenomenex) with solvent A (0.1\% acetic acid/water) and solvent B ( $0.1 \%$ acetic acid/acetonitrile). The column was eluted with $30 \%$ B for 5 minute followed by a linear gradient to $40 \% \mathrm{~B}$ over 15 minute and then increasing linearly to $90 \% \mathrm{~B}$ over 15 minute at a flow rate of $0.3 \mathrm{ml} / \mathrm{min}$. The column effluent was directed into the ESI source of a LCQ mass spectrometer (Thermo Fisher Scientific). The ESI conditions were: sheath gas flow rate, 90 arbitrary units; auxiliary gas, 30 arbitrary units; spray voltage, $4.5 \mathrm{kV}$; capillary temperature, $170^{\circ} \mathrm{C}$; capillary voltage, $30 \mathrm{~V}$; and tube lens offset, $25 \mathrm{~V}$. Data were acquired in positive ion mode using Xcalibur software (Thermo Fisher Scientific) with one full scan followed by two data-dependent scans of the most intense and the second most intense ions.

\section{Results}

OHEB Carboxylation Activity. The chemical structures of the three acetylenic compounds: MS, OD, and DZ as well as the EB prodrug, the OHEB intermediate metabolite, and the active CEB metabolite are shown in Fig. 1. Since EB hydroxylation to OHEB is primarily catalyzed by CYP2J2, the metabolism of EB was initially characterized in the CYP2J2 reconstituted systems (Hashizume et al., 2002). As shown in 

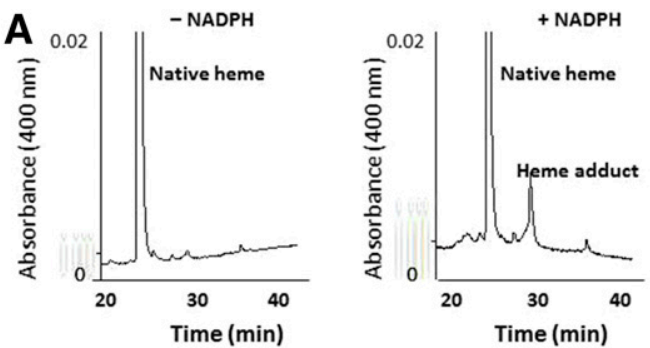

B

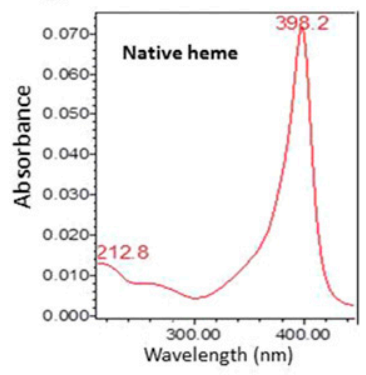

C

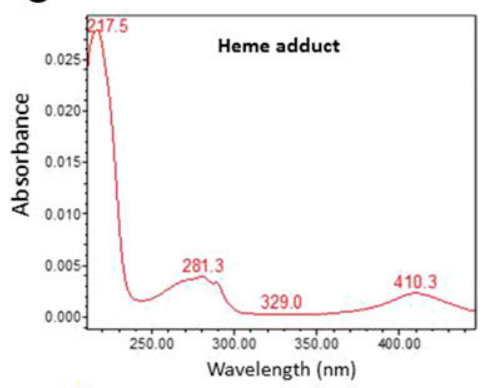

D

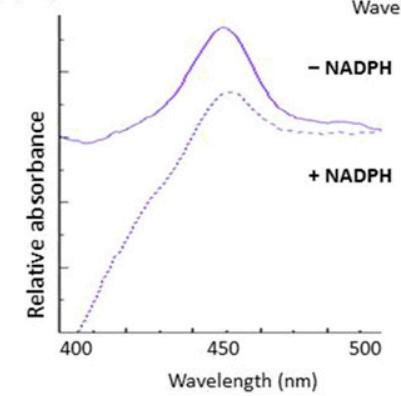

Fig. 4. Modification of the heme prosthetic group of CYP2J2 by MS. (A) HPLC elution profiles monitored at $400 \mathrm{~nm}$ for prosthetic heme in the absence or presence of NADPH. Native heme loss and heme adduct formation were observed in the +NADPH sample. (B) Photodiode-array spectrum of the native heme eluting at 26 minute. (C) Photodiode-array spectrum of the heme adduct eluting at 30 minute. (D) The reduced CO difference spectrum of the reconstituted system incubated with MS in the absence and presence of NADPH. The experimental procedures are described under Materials and Methods.

Fig. 2A, roughly $10 \%$ of initial OHEB produced was further metabolized to give $\mathrm{CEB}$ in a 5 minute incubation. Therefore, $\mathrm{OHEB}$ was chosen as the substrate probe to characterize the catalytic activity and kinetics of CYP2J2 to avoid confounding analyses due to sequential hydroxylation (Hashizume et al., 2002; Liu et al., 2006). As shown in Fig. 2B, the formation of $\mathrm{CEB}$ from $\mathrm{OHEB}$ at concentrations ranging from 0.1 to $40 \mu \mathrm{M}$ was determined and the results fitted to the Michaelis-Menten equation. The $K_{\mathrm{m}}$ is $3.8 \mu \mathrm{M}$ and the $k_{\text {cat }}$ is $8.8 \mathrm{~min}^{-1}$ for the carboxylation of OHEB to CEB. The abilities of CYP2J2 and CYP3A4 in the reconstituted systems to metabolize OHEB to CEB were compared with confirm that CYP2J2, but not CYP3A4, is the P450 that forms the CEB active metabolite (Fig. 2C).

Kinetic Values for the Inactivation of CYP2J2 by MS. The inactivation of the CYP2J2-catalyzed carboxylation of OHEB by MS was measured using various concentrations of MS (1-200 $\mu \mathrm{M})$ at different time points $(4,8,12)$ for each concentration. As shown in Fig. $3 \mathrm{~A}$, the inactivation of CYP2J2 required NADPH and was time- and MS concentration-dependent. Linear regression analysis of the time course data were used to estimate the initial rate constants $\left(k_{\text {obs }}\right)$ for the inactivation of CYP2J 2 by the various concentrations of MS. The kinetic values were obtained by fitting the $k_{\text {obs }}$ values at the various concentrations of MS to the Michaelis-Menten equation. The $K_{\mathrm{I}}$ and $k_{\text {inact }}$ were determined to be $6.1 \mu \mathrm{M}$ and $0.22 \mathrm{~min}^{-1}$, respectively (Fig. 3B).
Partition Ratio for the Inactivation of CYP2J2 by MS. CYP2J2 was incubated with varying concentrations of MS for 30 minute in order for the inactivation to reach completion. The percentage of activity remaining was plotted as a function of the molar ratio of the inactivator to CYP450 (Fig. 3C). The partition ratio was estimated from the intercept of the linear regression lines obtained from the lower ratios of MS to CYP450 with the straight line derived from higher ratios of MS to CYP450. Using this method, the partition ratio for the inactivation of CYP2J2 by MS was estimated to be $\sim 10$.

Heme Modification Following Inactivation of CYP2J2 by MS. In the absence (control) or presence (inactivated) of NADPH, CYP2J2containing reaction mixtures were incubated with $50 \mu \mathrm{M}$ MS for 10 minute and analyzed by HPLC to determine the nature and extent of heme modification. Monitoring for eluates at $400 \mathrm{~nm}$, the MSinactivated sample had a $65 \%$ decrease in prosthetic heme (26 minute) coupled with the formation of a heme adduct (30 minute) as compared with the control sample (Fig. 4A). Analysis of the two peaks using a photodiode-array detector coupled to the HPLC showed that the wavelength for the maximal absorption spectrum of native heme eluting at 26 minute was $398 \mathrm{~nm}$ (Fig. 4B), whereas the wavelength for maximal absorption spectrum of modified heme eluting at 30 minute was $410 \mathrm{~nm}$. The absorbance at $217 \mathrm{~nm}$ was most likely due to the presence of the inactivator MS (Fig. 4C). This result suggests that the reactive metabolite of MS covalently binds to prosthetic heme of CYP2J2. Under the same conditions, the spectrally detectable reduced $\mathrm{CO}$ complex of the MS-inactivated sample was approximately $36 \%$ of the control sample (Fig. 4D).

Mechanism-Based Inactivation of CYP2J2 by OD. Following the same procedures previously described for MS, the time- and OD concentration-dependent loss of OHEB carboxylation activity was characterized, see Fig. 5A. The $K_{\mathrm{I}}$ and $k_{\text {inact }}$ were determined to be $2.5 \mu \mathrm{M}$ and $0.05 \mathrm{~min}^{-1}$, respectively (Fig. 5B). The partition ratio for OD was estimated to be $\sim 20$ (Fig. 5C). When CYP2J2 was incubated with $50 \mu \mathrm{M} \mathrm{OD}$ at $37^{\circ} \mathrm{C}$ for 10 minute, a $50 \%$ loss was observed in native heme (Fig. 6A) and the spectrally detectable reduced $\mathrm{CO}$ spectrum (Fig. 6B) for the inactivated sample when compared with the control sample. Unlike inactivation by MS, no modified heme was observed following inactivation by OD (data not shown).

LC-MS/MS Analysis of the GSH Conjugates Formed by Metabolism of MS or OD by CYP2J2. To identify the reactive intermediates formed during the metabolism of MS or OD by CYP2J2, incubations were performed in the presence of $2 \mathrm{mM} \mathrm{GSH}$ and the GSH conjugates were characterized by LC-MS/MS analysis as described in Materials and Methods. The full mass spectrum of the MS-GSH conjugate with $m / z 647$ is equivalent to the mass of GSH, MS and one oxygen atom (Fig. 7A). The MS/MS spectrum of this conjugate is displayed in Fig. 7B. The major fragment ion at $\mathrm{m} / z, 572$ was from the loss of Gly (75 Da) and the fragment ion at $\mathrm{m} / z, 500$ was from loss of Glu (129 Da) and water. The fragment ion at $\mathrm{m} / \mathrm{z}, 397$ was from the combination loss of Gly, Glu, $\mathrm{C}=\mathrm{O}$, and water. All of these fragment ions indicate that GSH is a component of this MS-GSH conjugate (Baillie and Davis, 1993). The fragment ion at $\mathrm{m} / \mathrm{z}, 552$ is from the cleavage of the amide bond with the methylsulfonyl moiety side chain and water. The subsequent loss of Gly forms a fragment ion at $m / z 477$. Cleavage of the C-C bond next to the carbonyl moiety of MS forms the fragment ion at $\mathrm{m} / \mathrm{z}, 322$. The fragment ion at $\mathrm{m} / \mathrm{z}, 215$, a component of GSH, represents the combined loss of Gly and water. The proposed structure of the MS-GSH conjugate is shown in Fig. 7C. The sites of MS/MS fragmentation are indicated by the dashed lines.

The full mass spectrum of the OD-GSH conjugate with $\mathrm{m} / \mathrm{z} 603$ is shown in Fig. 8A and the MS/MS spectrum of this conjugate is displayed in Fig. 8B. The major fragment ion at $m / z 528$ was from the loss of Gly 


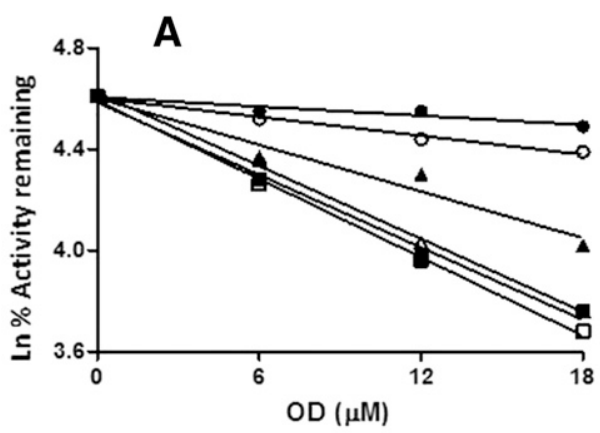

B

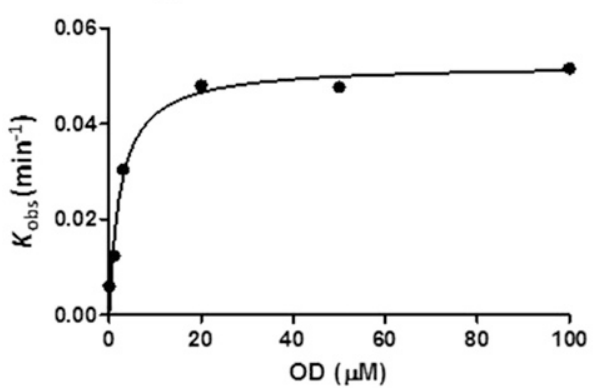

C

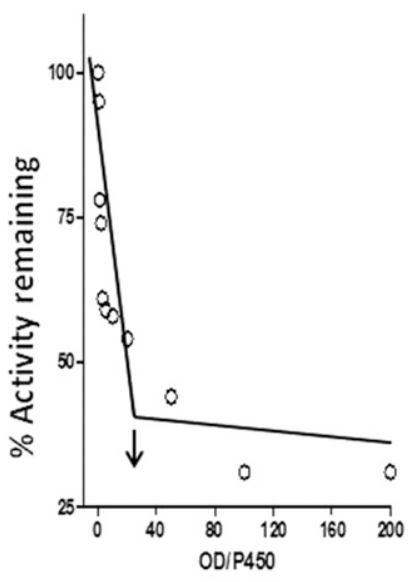

Fig. 5. Time- and concentration-dependent loss of OHEB carboxylation and determination of the partition ratio for CYP2J2 during inactivation by OD. (A) CYP2J2 in the reconstituted system was incubated with $0(\bullet), 1(\bigcirc), 3(\boldsymbol{\Delta}), 20(\Delta), 50(\mathbf{\square})$, and $100 \mu \mathrm{M}(\square)$ $\mathrm{OD}$ and aliquots were removed at the times indicated and assayed for residual OHEB metabolism as described under Materials and Methods. The catalytic activity at time zero was used as the $100 \%$ control to calculate the initial rate constants for the inactivation $\left(k_{\text {obs }}\right)$ for each concentration of OD. (B) Shows the fitting of the initial rate constants for the inactivation as a function of the OD concentrations to the Michaelis-Menten equation. The $K_{\mathrm{I}}$ and $k_{\text {inact }}$ values were determined to be $2.5 \mu \mathrm{M}$ and $0.05 \mathrm{~min}^{-1}$, respectively. (C) Determination of the partition ratio for the inactivation of CYP2J2 by OD. The percentage of catalytic activity remaining was determined as a function of the molar ratio of OD to CYP2J2 as described under Materials and Methods. The partition ratio $(\sim 20)$ was estimated from the intercept of the linear regression line from the lower ratios of OD to CYP2J2 and the straight line obtained from higher ratios of OD to CYP2J2. The data represent the average of two separate experiments.
(75 Da), the fragment ion at $m / z 474$ was from loss of Glu (129 Da), and the subsequent loss of water leads to formation of the ion at $m / z 456$. The fragment ion at $\mathrm{m} / \mathrm{z} 371$ originated from the combination loss of Gly, Glu, and $\mathrm{C}=\mathrm{O}$. All of these fragment ions indicate that GSH is a component of the OD-GSH conjugate (Baillie and Davis, 1993). Cleavage of the C-S bond forms the protonated GSH $(\mathrm{m} / \mathrm{z}, 308)$ and oxygenated OD $(\mathrm{m} / \mathrm{z}, 296)$. The proposed structure of the OD-GSH conjugate is shown in Fig. 8C. The sites for the MS/MS fragmentation are indicated by the dashed lines.
Difference Spectrum for the Binding of DZ to CYP2J2. The difference absorbance spectra for the addition of increasing amounts of DZ ( $4 \mathrm{nM}$ to $4 \mu \mathrm{M}$ ) to CYP2J2 are displayed in Fig. 9A. These difference spectra displayed peaks at $\sim 428 \mathrm{~nm}$ and troughs at $\sim 407 \mathrm{~nm}$. The $K_{\mathrm{s}}$ for the binding of DZ to CYP2J 2 was determined to be $2.8 \mu \mathrm{M}$ by fitting the data for the difference between the absorbance at 428 and 407 at each concentration of DZ as described in Materials and Methods, see Fig. 9A inset. The data obtained from the inhibition of OHEB carboxylation by DZ were fitted to the $\log$ [inhibitor] versus response
A

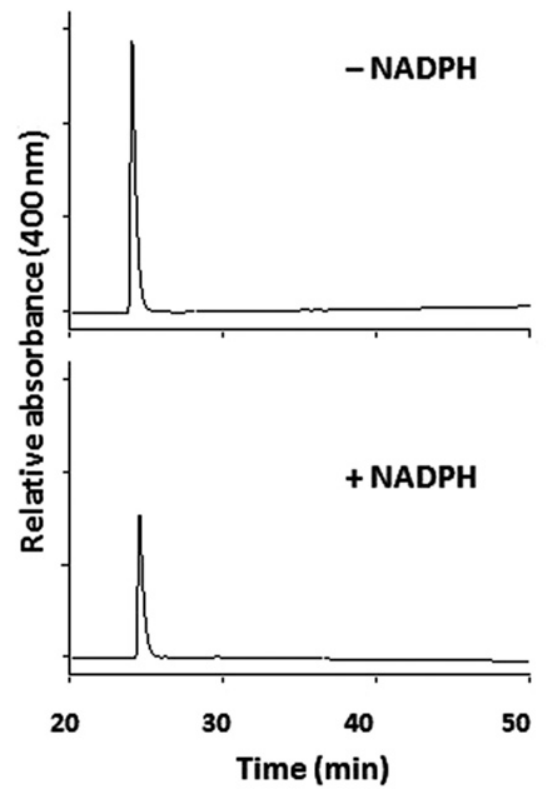

B

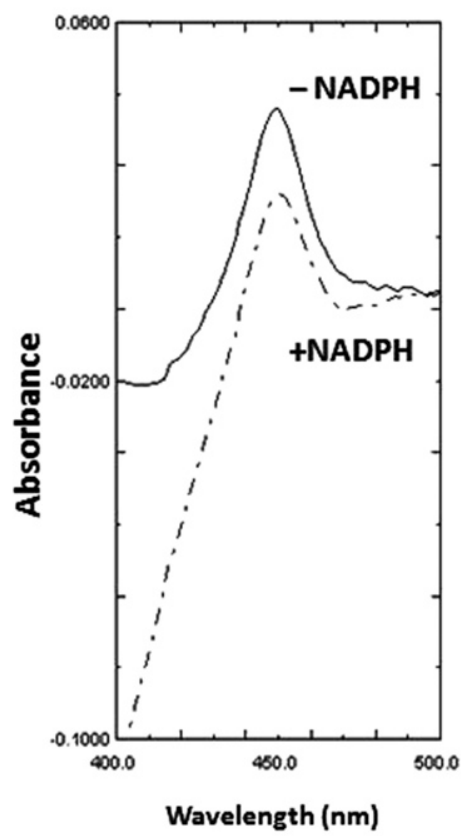

Fig. 6. Change in the CYP2J2 native heme due to the inactivation by OD. (A) HPLC elution profiles monitored at $400 \mathrm{~nm}$ for the prosthetic heme in the -NADPH and +NADPH samples. (B) The reduced CO difference spectra of the reconstituted system incubated with OD in the absence and presence of NADPH. The experimental procedures are described under Materials and Methods. 
A Full mass

$m / z 647$
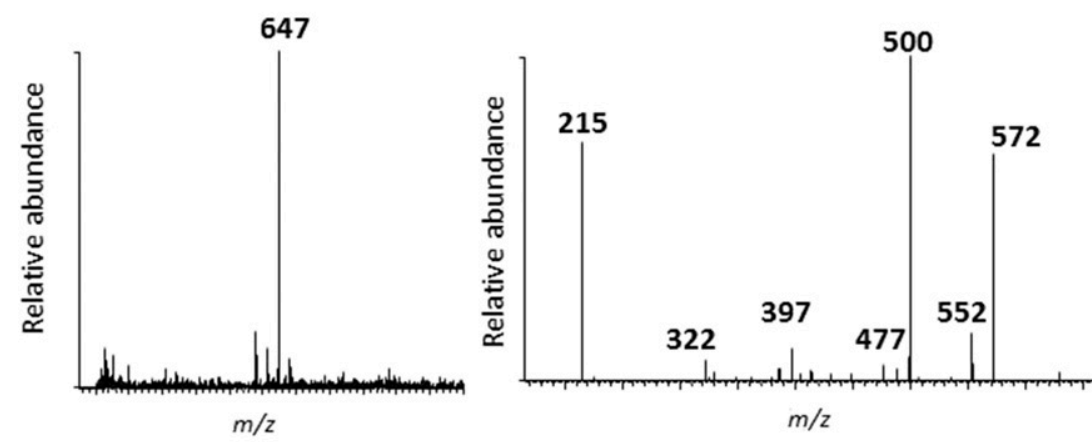

C MS-GSH conjugate structure

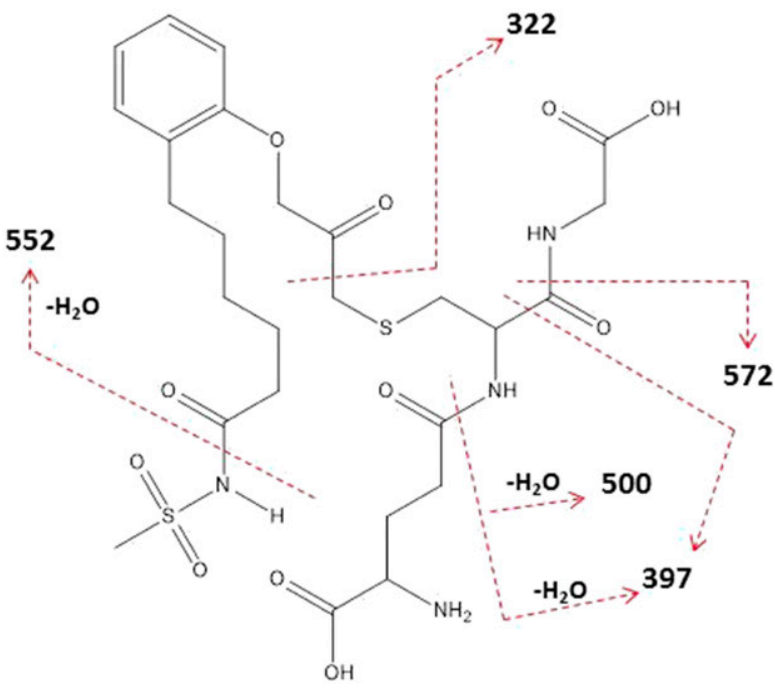

Fig. 7. LC-MS/MS analysis of the GSH conjugate formed during the metabolism of MS. A reaction mixture containing CYP2J2 was incubated with MS in the presence of NADPH and the reactive intermediate was trapped with $10 \mathrm{mM} \mathrm{GSH}$. The GSH conjugate was analyzed by LC-MS as described under Materials and Methods. (A) The full mass spectrum of the MS-GSH conjugate with the $\mathrm{MH}^{+}$ion at $m / z$ 647. (B) The MS/MS spectrum of the MS-GSH conjugate. (C) The proposed structure of the MS-GSH conjugate. The dashed lines indicate the sites of fragmentation. The MS/MS spectrum was obtained in the positive mode and analyzed using the Xcalibur software package. equation (GraphPad Prism5 software) to calculate the $\mathrm{IC}_{50}$ value of $0.18 \mu \mathrm{M}$ for DZ (Fig. 9B).

\section{Discussion}

CYP2J2 is highly expressed in heart and vascular tissues. It is particularly abundant in endothelial cells and it is active in the epoxidation of arachidonic acid to EETs which have potent vasodilatory, antiflammatory, and cardioprotective properties (Wu et al., 1996; Node et al., 1999; Yang et al., 2001; Chaudhary et al., 2009). Additionally, in human intestine, CYP2J2 plays a critical role in the first-pass metabolism of therapeutic drugs, particularly the antihistamines astemizole and EB to pharmacologically active metabolites desmethylastemizole and CEB, respectively (Zeldin et al., 1997; Hashizume et al., 2002; Matsumoto et al., 2002; Liu et al., 2006). Recently, a number of substrates and inhibitors of CYP2J2 have been identified (Lee et al., 2010, 2012; Ren et al., 2013). The latest guidance from the US Food and Drug Administration on industrial drug studies suggests that CYP2J2 should be considered in the metabolism of all new drugs (US Department of Health and Human Services, Food and Drug Administration (FDA), Center for Drug Evaluation and Research (CDER). Center for Biologics Evaluation and Research (CBER), 2012). Because of the important role of CYP2J2 in cardiovascular regulation and in drug metabolism, it is necessary to expand our understanding of its mechanism-based inactivation by clinical drugs and herbal supplements. With its wide range of tissue distribution, it is possible that CYP2J2 can cause drug-drug and food-drug interactions in extrahepatic tissue, particularly in the gastrointestinal tract (Zeldin et al., 1997; Hashizume et al., 2002; Matsumoto et al., 2002; Lee et al., 2012; Ren et al., 2013). Thus, further studies in the inhibition and inactivation of CYP2J2 are warranted.

It has previously been demonstrated that the dealkylation of $\mathrm{EB}$ and its metabolites are mainly catalyzed by CYP3A4, whereas the hydroxylation of EB and its metabolite are preferentially catalyzed by CYP2J2 (Hashizume et al., 2002; Liu et al., 2006). During the course of this study, it was found that OHEB, the primary metabolite of EB catalyzed by $\mathrm{CYP} 2 \mathrm{~J} 2$, is readily converted to CEB by CYP $2 \mathrm{~J} 2$ in the reconstituted system. For comparison, the kinetics for the formation of the first metabolite from EB and OHEB by CYP2J2 were determined and the data analyzed using the Michaelis-Menten equation. The $K_{\mathrm{m}}$ is $23 \mu \mathrm{M}$ and $k_{\text {cat }}$ is $20 \mathrm{~min}^{-1}$ for the formation of OHEB from EB (data not shown). The efficiency $\left(k_{\mathrm{cat}} / K_{\mathrm{m}}\right)$ for the EB hydroxylation to form OHEB is $0.86 \mathrm{~min}^{-1} \mu \mathrm{M}^{-1}$, whereas the efficiency for the OHEB carboxylation is $2.32 \mathrm{~min}^{-1} \mu \mathrm{M}^{-1}$ (data obtained from Fig. 2B), indicating that metabolism of EB to OHEB is significantly less efficient than the conversion of OHEB to CEB. Moreover, CEB formation by 
A Full mass
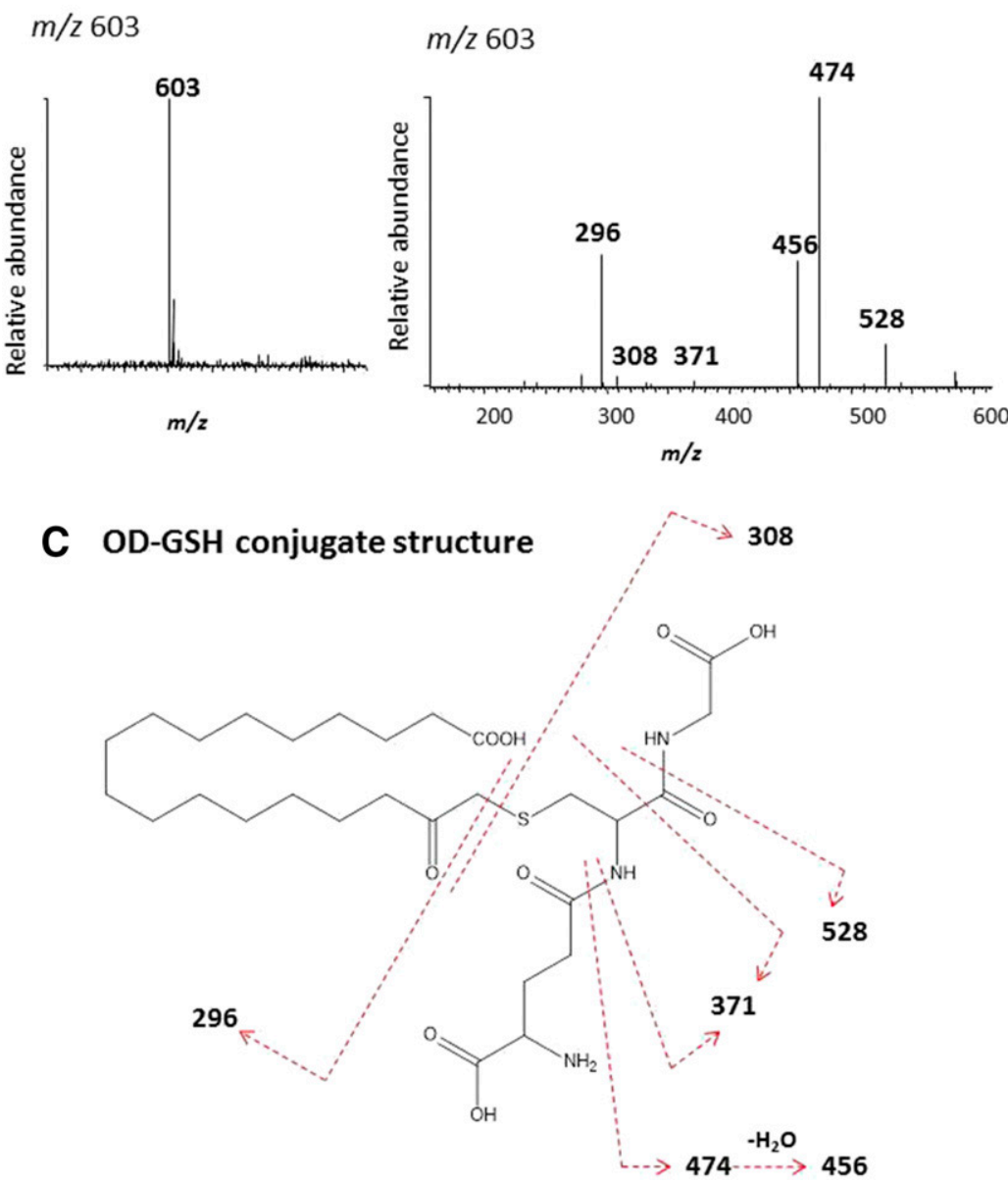

Fig. 8. LC-MS/MS analysis of the GSH conjugate formed during the metabolism of OD. A reaction mixture containing CYP2J2 was incubated with OD in the presence of NADPH and the reactive intermediate was trapped with $2 \mathrm{mM} \mathrm{GSH}$. The GSH conjugate was analyzed by LC-MS as described under Materials and Methods. (A) The full mass spectrum of the OD-GSH conjugate with the $\mathrm{MH}^{+}$ion at $m / z$ 603. (B) The MS/MS spectrum of the OD-GSH conjugate. (C) The proposed structure of the OD-GSH conjugate. The dashed lines indicate the sites of fragmentation. The MS/MS spectrum was obtained in the positive mode and analyzed using the Xcalibur software package.
CYP3A4 is negligible when compared with that catalyzed by CYP2J2 (Fig. 2C). Our results reported here further support that the sequential metabolism of EB to OHEB and further conversion of OHEB to CEB is specific for CYP2J2.

Because MS and OD are mechanism-based inactivators, the apparent $K_{\mathrm{I}}, k_{\text {inact }}$, partition ratio, and general mechanism of action were determined for these compounds. For MS, the apparent $K_{\mathrm{I}}$ is $6.1 \mu \mathrm{M}$, the $k_{\text {inact }}$ is $0.22 \mathrm{~min}^{-1}$, and the partition ratio is $\sim 10$. For OD, the apparent $K_{\mathrm{I}}$ is $2.5 \mu \mathrm{M}$, the $k_{\text {inact }}$ is $0.05 \mathrm{~min}^{-1}$, and the partition ratio is $\sim 20$. The loss of OHEB carboxylation activity following incubation of either MS or OD correlated well with the loss of prosthetic heme and the decrease in the spectrally detectable reduced $\mathrm{CO}$ complex. Additionally, the inactivation of CYP2J2 by MS resulted in the formation of a dissociable adducted heme, indicating that a reactive intermediate of MS covalently bound to the prosthetic heme group. However, the heme adduct was not abundant and the mass of the reactive intermediate forming the adducted heme and contributing to the mechanism-based inactivation could not be determined by MS analysis of the modified heme.

In most the cases, the mass and the structure of the reactive intermediate binding to the heme or apoprotein can be deduced from the identity of the GSH conjugate resulting from the reaction between GSH and the reactive moiety of the inactivator formed during metabolism by the CYP450. For example, the covalent binding of ethynyl compounds to the prosthetic heme moiety and/or to the apoprotein have been previously shown with a variety of CYP450s including 1A1, 2B1, 2B6, 3A4 and 3A5 (Ortiz de Montellano and Komives, 1985; Chan et al.,
1993; Lin et al., 2002, 2009, 2011; Kent et al., 2006; Lin and Hollenberg, 2007). It has been reported that if the activated oxygen atom is added to the internal carbon of the ethynyl group, the heme will be alkylated. On the other hand, if the activated oxygen is added to the terminal carbon of the ethynyl group, the protein will be modified (Ortiz de Montellano and Komives, 1985; CaJacob et al., 1988; Chan et al., 1993). Since the structures of MS- and OD-GSH conjugates have the oxygen attached to internal carbon based on the MS/MS fragmentation patterns (Figs. 7C and 8C, respectively), the primary mechanism for the inactivation of CYP2J2 by MS and OD appears to involve modification of the heme. Our results are consistent with oxygen addition to the internal carbon of acetylene group. We believe that the reactive intermediate covalently bound to GSH is the same species that is responsible for the mechanism of the irreversible inactivation of CYP2J2 by MS and OD. Thus, we have characterized in detail the molecular mechanisms responsible for the irreversible inactivation of CYP2J2 by MS and OD in the reconstituted system. Two derivatives of the drugs, terfenadine and ebastine (EB), have been synthesized and identified as mechanism-based inhibitors of CYP2J2 expressed in insect cell microsomes. However, unlike MS and OD, the functional groups of these two derivatives are a carbon-carbon double bond and a benzodioxolederived carbene complex, respectively (Lafite et al., 2007a), rather than acetylene groups.

Although DZ is a terminal acetylenic compound and might be expected to lead to mechanism-based inactivation, no evidence of inactivation of CYP2J2 was observed. In contrast, DZ is a very efficient mechanism-based inactivator of CYP3A4 (H. L. Lin and P. F. 


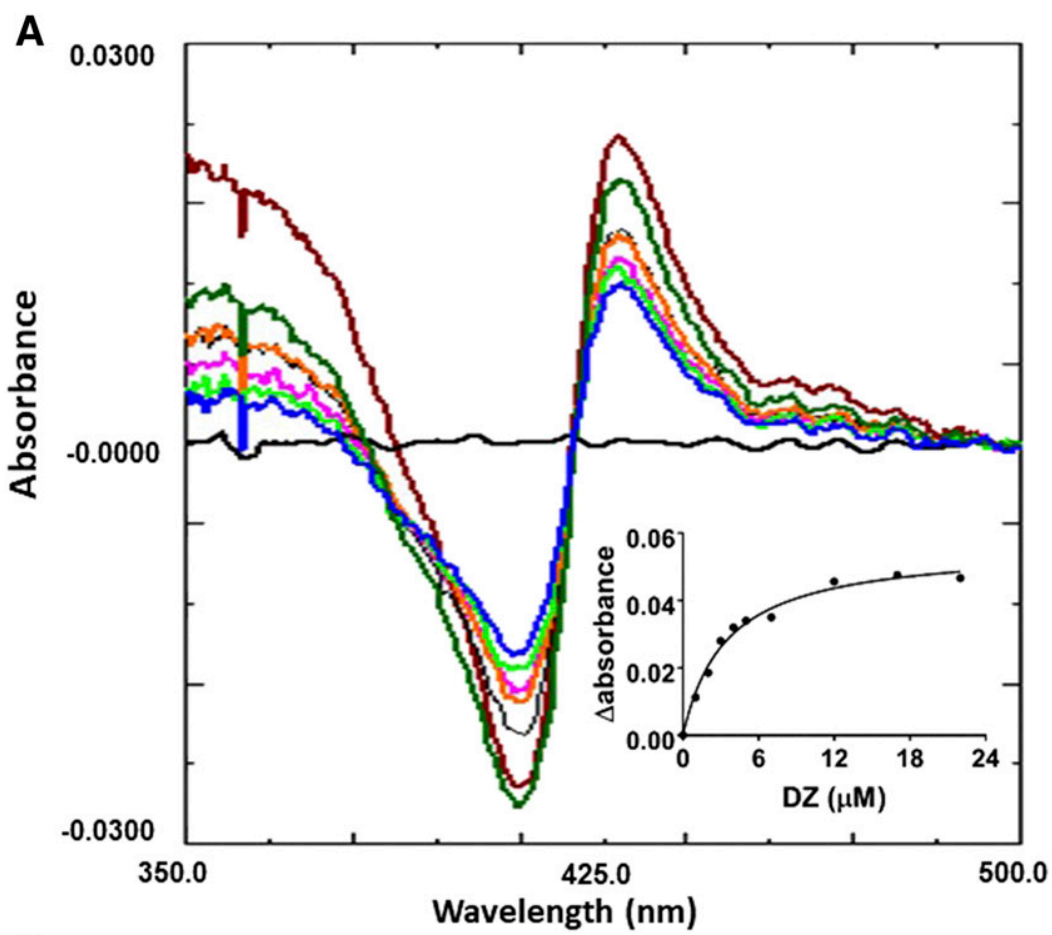

B

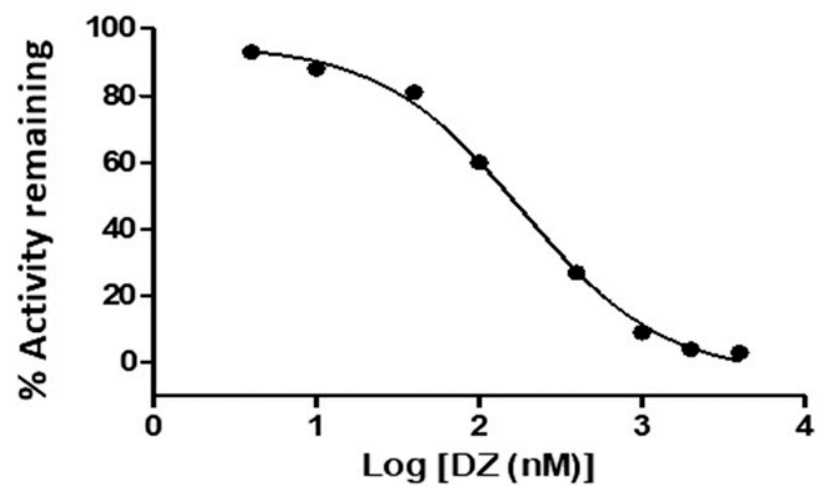

Fig. 9. Optical spectra for the binding of DZ to CYP2J2. (A) DZ binding to CYP2J2 was performed as described in Materials and Methods and resulted in a type II binding spectrum as shown here. Inset, the difference in the absorbance between the peak and the trough of each spectrum plotted versus the corresponding DZ concentration. The data points were fitted to the Michaelis-Menten equation. The $K_{\mathrm{s}}$ was estimated to be $2.8 \mu \mathrm{M}$ DZ. (B) Determination of the $\mathrm{IC}_{50}$ for $\mathrm{DZ}$ inhibition of the carboxylation of $20 \mu \mathrm{M}$ OHEB as described in Materials and Methods. The data were fitted to the dose-response equation as described under Materials and Methods.
Hollenberg, unpublished observations). These results suggest that bioactivation of the carbon-carbon triple bond in DZ was catalyzed during the metabolism of DZ by CYP3A4, but not by CYP2J2. For comparison, the $K_{\mathrm{s}}$ value for type II binding of DZ to CYP3A4 was determined to be $10 \mu \mathrm{M}$ (data not shown), which is about fourfold higher than the $K_{\mathrm{s}}$ value for binding to CYP2J2 $(2.8 \mu \mathrm{M})$. This indicates that the affinity of isoxazole moiety to the heme iron in CYP2J2 is stronger than it is for CYP3A4. Our findings are in agreement with previous observations that incubation of $\mathrm{DZ}$ with $\mathrm{CYP} 2 \mathrm{~J} 2$ resulted in the oxidative cleavage of the isoxazole moiety (Lee et al., 2010). These data conflict with what has been observed with CYP3A4. CYP3A4 metabolizes DZ at multiple sites because of its ability to rotate freely in the active site (Lee et al., 2010). Different from the active site of CYP3A4, CYP2J2 has a narrow hydrophobic channel that restricts access to the heme (Lafite et al., 2007b). It is likely that the ethynyl moiety of DZ is not oriented close to the heme iron of CYP2J2 thus preventing the formation of a reactive intermediate for mechanism-based inactivation.

Danazol was previously demonstrated as a potent inhibitor of CYP2J2-catalyzed reactions including amiodarone 4-hydroxylation and astemizole $O$-demethylation (Lee et al., 2012). Here, we report that the $\mathrm{IC}_{50}$ of $\mathrm{DZ}$ for inhibition of OHEB metabolism is $<0.2 \mu \mathrm{M}$. Moreover, our laboratory has observed that $0.1 \mu \mathrm{M}$ DZ and OD $50 \mu \mathrm{M}$ can inhibit the metabolism of arachidonoyl ethanolamide (an endogenous amide of AA) to similar extents (V. J. Walker and P. F. Hollenberg, unpublished observation). In short, DZ is a potent and specific inhibitor of CYP2J2 that can be used in conjunction with substrate probes, including OHEB, amiodarone, and astemizole, to study the role of CYP2J2 in liver and intestinal microsomes or other tissue preparations (Lee et al., 2012).

In this study, we investigated the mode of inhibition of three widely used selective inhibitors of CYP2J2: MS, OD and DZ. OD has previously been used to investigate the effect of CYP2J2 epoxygenase on the proliferation of tumor cells (Jiang et al., 2005, 2009). Likewise, MS was used to evaluate protection by EETs against tumor necrosis factor- $\alpha$-induced injury in rat hearts and cell culture and the inhibition of carcinoma cell motility (Nithipatikom et al., 2010; Zhao et al., 2012). Although DZ also inhibits CYPs 3A4, 2C8, 2 C9 and 2D6, the $\mathrm{IC}_{50}$ value for CYP2J2 inhibition is much lower. As a result, DZ was used to determine the metabolic role of CYP2J2 in hepatic and extrahepatic tissues (Lee et al., 2012). Here, we report that MS and OD, but not DZ, inhibit this CYP450 by acting as mechanism-based inactivators of 
CYP2J2. Differences in mechanisms of inhibition are important physiologically since activity loss due to mechanism-based inactivation is not reversible and metabolic activity can only be regained following synthesis of more $2 \mathrm{~J} 2$. For reversible inhibition, there is no inactivation and metabolic activity can be regained more rapidly since the concentration of the effected drug increases and thus can compete more effectively for metabolism.

\section{Acknowledgments}

We thank Dagan K. Hammar for the purification of CYP2J2.

\section{Authorship Contributions}

Participated in research design: Lin, Walker, D'Agostino.

Conducted experiments: Lin, Walker.

Contributed new reagents or analytical tools: Lin, D'Agostino.

Performed data analysis: Lin, Zhang, Walker, D'Agostino.

Wrote or contributed to writing of manuscript: Lin, Zhang, Walker, Hollenberg.

\section{References}

Baillie TA and Davis MR (1993) Mass spectrometry in the analysis of glutathione conjugates. Biol Mass Spectrom 22:319-325.

Brand-Schieber E, Falck JF, and Schwartzman M (2000) Selective inhibition of arachidonic acid epoxidation in vivo. $J$ Physiol Pharmacol 51:655-672.

CaJacob CA, Chan WK, Shephard E, and Ortiz de Montellano PR (1988) The catalytic site of rat hepatic lauric acid omega-hydroxylase. Protein versus prosthetic heme alkylation in the omegahydroxylation of acetylenic fatty acids. J Biol Chem 263:18640-18649.

Chan WK, Sui Z, and Ortiz de Montellano PR (1993) Determinants of protein modification versus heme alkylation: inactivation of cytochrome $\mathrm{P} 4501 \mathrm{~A} 1$ by 1-ethynylpyrene and phenylacetylene. Chem Res Toxicol 6:38-45.

Chaudhary KR, Batchu SN, and Seubert JM (2009) Cytochrome P450 enzymes and the heart IUBMB Life 61:954-960.

Chen C, Li G, Liao W, Wu J, Liu L, Ma D, Zhou J, Elbekai RH, Edin ML, Zeldin DC, et al. (2009) Selective inhibitors of CYP2J2 related to terfenadine exhibit strong activity against human cancers in vitro and in vivo. J Pharmacol Exp Ther 329:908-918.

Dutheil F, Dauchy S, Diry M, Sazdovitch V, Cloarec O, Mellottée L, Bièche I, Ingelman-Sundberg M, Flinois JP, de Waziers I, et al. (2009) Xenobiotic-metabolizing enzymes and transporters in the normal human brain: regional and cellular mapping as a basis for putative roles in cerebral function. Drug Metab Dispos 37:1528-1538.

Fleming I and Busse R (2006) Endothelium-derived epoxyeicosatrienoic acids and vascular function. Hypertension 47:629-633.

Hashizume T, Imaoka S, Mise M, Terauchi Y, Fujii T, Miyazaki H, Kamataki T, and Funae Y (2002) Involvement of CYP2J2 and CYP4F12 in the metabolism of ebastine in human intestinal microsomes. J Pharmacol Exp Ther 300:298-304.

Hashizume T, Mise M, Terauchi Y, O L, Fujii T, Miyazaki H, and Inaba T (1998) N-Dealkylation and hydroxylation of ebastine by human liver cytochrome P450. Drug Metab Dispos 26: $566-571$.

Jenkins CM, Cedars A, and Gross RW (2009) Eicosanoid signalling pathways in the heart. Cardiovasc Res 82:240-249.

Jiang JG, Chen CL, Card JW, Yang S, Chen JX, Fu XN, Ning YG, Xiao X, Zeldin DC, and Wang DW (2005) Cytochrome P450 2J2 promotes the neoplastic phenotype of carcinoma cells and is up-regulated in human tumors. Cancer Res 65:4707-4715.

Jiang JG, Shen GF, Chen C, Fu XN, and Wang DW (2009) Effects of cytochrome P450 arachidonic acid epoxygenases on the proliferation of tumor cells. Chin J Cancer 28:14-19.

Kent UM, Lin HL, Mills DE, Regal KA, and Hollenberg PF (2006) Identification of 17- $\alpha-$ ethynylestradiol-modified active site peptides and glutathione conjugates formed during metabolism and inactivation of P450s 2B1 and 2B6. Chem Res Toxicol 19:279-287.

Lafite P, André F, Zeldin DC, Dansette PM, and Mansuy D (2007b) Unusual regioselectivity and active site topology of human cytochrome P450 2J2. Biochemistry 46:10237-10247.

Lafite P, Dijols S, Buisson D, Macherey AC, Zeldin DC, Dansette PM, and Mansuy D (2006) Design and synthesis of selective, high-affinity inhibitors of human cytochrome P450 $2 \mathrm{~J} 2$. Bioorg Med Chem Lett 16:2777-2780.

Lafite P, Dijols S, Zeldin DC, Dansette PM, and Mansuy D (2007a) Selective, competitive and mechanism-based inhibitors of human cytochrome P450 2J2. Arch Biochem Biophys 464: $155-168$.

Lee CA, Jones, IIIJP, Katayama J, Kaspera R, Jiang Y, Freiwald S, Smith E, Walker GS, and Totah RA (2012) Identifying a selective substrate and inhibitor pair for the evaluation of CYP2J2 activity. Drug Metab Dispos 40:943-951.

Lee CA, Neul D, Clouser-Roche A, Dalvie D, Wester MR, Jiang Y, Jones, IIIJP, Freiwald S, Zientek M, and Totah RA (2010) Identification of novel substrates for human cytochrome P450 2J2. Drug Metab Dispos 38:347-356.

Lin HL, D'Agostino J, Kenaan C, Calinski D, and Hollenberg PF (2013) The effect of ritonavir on human CYP2B6 catalytic activity: heme modification contributes to the mechanism-based inactivation of CYP2B6 and CYP3A4 by ritonavir. Drug Metab Dispos 41:1813-1824.
Lin HL and Hollenberg PF (2007) The inactivation of cytochrome P450 3A5 by $17 \alpha$-ethynylestradiol is cytochrome $b_{5}$-dependent: metabolic activation of the ethynyl moiety leads to the formation of glutathione conjugates, a heme adduct, and covalent binding to the apoprotein. J Pharmacol Exp Ther 321:276-287.

Lin HL, Kent UM, and Hollenberg PF (2002) Mechanism-based inactivation of cytochrome P450 3 A4 by $17 \alpha$-ethynylestradiol: evidence for heme destruction and covalent binding to protein. $J$ Pharmacol Exp Ther 301:160-167.

Lin HL, Zhang H, Noon KR, and Hollenberg PF (2009) Mechanism-based inactivation of CYP2B1 and its F-helix mutant by two tert-butyl acetylenic compounds: covalent modification of prosthetic heme versus apoprotein. J Pharmacol Exp Ther 331:392-403.

Lin HL, Zhang H, Pratt-Hyatt MJ, and Hollenberg PF (2011) Thr302 is the site for the covalent modification of human cytochrome P450 2B6 leading to mechanism-based inactivation by tertbutyl phenylacetylene. Drug Metab Dispos 39:2431-2439.

Liu KH, Kim MG, Lee DJ, Yoon YJ, Kim MJ, Shon JH, Choi CS, Choi YK, Desta Z, and Shin JG (2006) Characterization of ebastine, hydroxyebastine, and carebastine metabolism by human liver microsomes and expressed cytochrome P450 enzymes: major roles for CYP2J2 and CYP3A. Drug Metab Dispos 34:1793-1797.

Matsumoto S, Hirama T, Matsubara T, Nagata K, and Yamazoe Y (2002) Involvement of CYP2J2 on the intestinal first-pass metabolism of antihistamine drug, astemizole. Drug Metab Dispos 30: $1240-1245$.

Nithipatikom K, Brody DM, Tang AT, Manthati VL, Falck JR, Williams CL, and Campbell WB (2010) Inhibition of carcinoma cell motility by epoxyeicosatrienoic acid (EET) antagonists. Cancer Sci 101:2629-2636.

Node K, Huo Y, Ruan X, Yang B, Spiecker M, Ley K, Zeldin DC, and Liao JK (1999) Antiinflammatory properties of cytochrome P450 epoxygenase-derived eicosanoids. Science $\mathbf{2 8 5}$. $1276-1279$.

Ortiz de Montellano PR and Komives EA (1985) Branchpoint for heme alkylation and metabolite formation in the oxidation of arylacetylenes by cytochrome P-450. J Biol Chem 260:3330-3336.

Ren S, Zeng J, Mei Y, Zhang JZH, Yan SF, Fei J, and Chen L (2013) Discovery and characterization of novel, potent, and selective cytochrome P450 2J2 inhibitors. Drug Metab Dispos 41: $60-71$.

Scott EE, Spatzenegger M, and Halpert JR (2001) A truncation of 2B subfamily cytochromes P450 yields increased expression levels, increased solubility, and decreased aggregation while retaining function. Arch Biochem Biophys 395:57-68.

Seubert J, Yang B, Bradbury JA, Graves J, Degraff LM, Gabel S, Gooch R, Foley J, Newman J, Mao L, et al. (2004) Enhanced postischemic functional recovery in CYP2J2 transgenic hearts involves mitochondrial ATP-sensitive $\mathrm{K}^{+}$channels and p42/p44 MAPK pathway. Circ Res 95:506-514.

Seubert JM, Zeldin DC, Nithipatikom K, and Gross GJ (2007) Role of epoxyeicosatrienoic acids in protecting the myocardium following ischemia/reperfusion injury. Prostaglandins Other Lipid Mediat 82:50-59.

Silverman R (1996) Mechanism-based enzyme inactivation, in Comtemporary Enzyme Kinetics and Mechanisms (Purich DL, ed) pp 291-335, Academic Press, San Diego, CA.

Smith HE, Jones, IIJJP, Kalhorn TF, Farin FM, Stapleton PL, Davis CL, Perkins JD, Blough DK, Hebert MF, Thummel KE, et al. (2008) Role of cytochrome P450 2C8 and 2J2 genotypes in calcineurin inhibitor-induced chronic kidney disease. Pharmacogenet Genomics 18:943-953.

Spector AA and Norris AW (2007) Action of epoxyeicosatrienoic acids on cellular function. Am J Physiol Cell Physiol 292:C996-C1012.

US Department of Health and Human Services, Food and Drug Administration (FDA), Center for Drug Evaluation and Research (CDER). Center for Biologics Evaluation and Research (CBER) (2012) Guidance for Industry, Drug Interaction Studies - Study Design, Data Analysis, Implication for Dosing, and Labeling Recommendations. Silver Spring, MD.

Wang MH, Brand-Schieber E, Zand BA, Nguyen X, Falck JR, Balu N, and Schwartzman ML (1998) Cytochrome P450-derived arachidonic acid metabolism in the rat kidney: characterization of selective inhibitors. J Pharmacol Exp Ther 284:966-973.

Wu S, Moomaw CR, Tomer KB, Falck JR, and Zeldin DC (1996) Molecular cloning and expression of CYP2J2, a human cytochrome $\mathrm{P} 450$ arachidonic acid epoxygenase highly expressed in heart. $J$ Biol Chem 271:3460-3468.

Wu Z, Lee D, Joo J, Shin JH, Kang W, Oh S, Lee DY, Lee SJ, Yea SS, Lee HS, et al. (2013) CYP2J2 and CYP2C19 are the major enzymes responsible for metabolism of albendazole and fenbendazole in human liver microsomes and recombinant P450 assay systems. Antimicrob Agents Chemother 57:5448-5456.

Yang B, Graham L, Dikalov S, Mason RP, Falck JR, Liao JK, and Zeldin DC (2001) Overexpression of cytochrome $\mathrm{P} 450 \mathrm{CYP} 2 \mathrm{~J} 2$ protects against hypoxia-reoxygenation injury in cultured bovine aortic endothelial cells. Mol Pharmacol 60:310-320.

Zeldin DC, Foley J, Goldsworthy SM, Cook ME, Boyle JE, Ma J, Moomaw CR, Tomer KB, Steenbergen C, and Wu S (1997) CYP2J subfamily cytochrome P450s in the gastrointestinal tract: expression, localization, and potential functional significance. Mol Pharmacol 51:931-943. Zeldin DC, Foley J, Ma J, Boyle JE, Pascual JMS, Moomaw CR, Tomer KB, Steenbergen C, and Wu S (1996) CYP2J subfamily P450s in the lung: expression, localization, and potential functional significance. Mol Pharmacol 50:1111-1117.

Zhao G, Wang J, Xu X, Jing Y, Tu L, Li X, Chen C, Cianflone K, Wang P, Dackor RT, et al (2012) Epoxyeicosatrienoic acids protect rat hearts against tumor necrosis factor- $\alpha$-induced injury. J Lipid Res 53:456-466.

Zhou AP, Ma YH, Sui ZH, Ortiz de Montellano PR, Clark JE, Masters BS, and Roman RJ (1994) Effect of 17-octadecynoic acid, a suicide-substrate inhibitor of cytochrome P450 fatty acid $\omega$-hydroxylase, on renal function in rats. J Pharmacol Exp Ther 268:474-481.

Address correspondence to: Paul F. Hollenberg, Department of Pharmacology, University of Michigan, 2220C MSRB III, 1150 West Medical Center Drive, Ann Arbor, MI 48109-5632. E-mail: phollen@umich.edu 